\title{
Reaping the fruits of collaboration - learning development research in the LearnHigher CETL network
}

\author{
Kim Shahabudin \\ University of Reading, UK
}

The LearnHigher CETL is now in its fourth year of operation, investigating bestpractice resources to support and enhance student learning development. LearnHigher is the largest collaborative CETL, pooling research and resources from learning and educational development professionals in 16 Higher Education institutions in England. [1] Through research into twenty core 'learning areas', LearnHigher partners have amassed a large quantity of data from students and staff. In 2008-09, a project was conducted to produce an overview of LearnHigher research so far, gathering insights on student preferences, needs and practices in learning areas including academic writing, critical thinking, doing research, group work and time management; on the use and design of learning spaces; and on the role of learning developers.[2] The rich picture produced by this review confirms the benefits of the network's collaborations between institutions, drawing together research conducted with diverse academic communities to reveal overarching trends and areas of interest in student learning development.

The strength of the LearnHigher network rests in its capacity to record and investigate a diversity of student experiences from a variety of institutional viewpoints, while retaining a single focus on learning development resources. This is made possible by the positioning of LearnHigher partners as learning and educational developers in a variety of staff and student facing roles, which enables them to interact with a wide cross-section of their university populations. Through this, LearnHigher is uniquely placed to obtain insights from a broadly constituted student community in higher education institutions across England. Collecting insights confirmed the importance of issues well known to and discussed among learning developers: for example, acculturation to academic practice, difficulties with structuring work, the preference for subject-embedded support. However, it also revealed new concerns including problems with synchronous and asynchronous 
chat, increasing demand for independent learning spaces, using and referencing new types of e-resources in research.

Partners collected research data using various methods. A number of formal research projects have been conducted on topics associated with the learning areas. However, the network is also committed to using practice-led enquiry, taking an action research approach to investigate interventions. This reflective approach to learning development practice has been especially fruitful, producing a wealth of information on student needs and preferences. It has also facilitated the valuing and incorporation of incidental insights, which have sometimes proved as significant as deliberately sought research data. The overview project aimed to capture the results of both formal research, and this more informally obtained data. Pooling such diverse types of evidence means that interpretations can be no more than indicative. However, the multi-faceted picture they build recommends this methodology as a fruitful way of accessing a broadly conceptualised student learning experience. It also offers a timely reminder that the embodied knowledge and expertise of learning and educational developers makes them invaluable resources in themselves.

One example of this cross-curricular viewpoint is found in data collected on referencing: one of the 20 learning areas used to structure research and resource development in the CETL. The Referencing learning area is co-ordinated the University of Bradford; research-informed outputs from this lead institution include a comprehensive web resource, a book aimed at a student readership (Neville, 2007) and a research project (currently underway) on student ideas about the relationship between referencing and academic writing. [3]

However, partners working on other learning areas have also collected data related to referencing. In the Assessment learning area, for instance, research has been undertaken on the use of plagiarism detection software in formative assessment tasks. Student self-development as independent thinkers is crucial in the learning area of Critical Thinking, and was observed to be related to understanding of referencing practices and the ability to use references as evidence to support arguments, rather than replace them. Many comments related to difficulties with practice that arise from incorrect expectations (students believing, for instance, that 
they 'know' how to use references at university because they have used them at school). This, in particular, reinforces the importance of the 'development', rather than the more finite 'skills', model in learning support.

Another example can be found in comments on the roles that different formats play in the effectiveness of resources. The CETL aims to make resources freely available through online dissemination via the LearnHigher website. However, subsequent delivery of these resources may be in electronic, paper or inter-personal format. LearnHigher research has already informed the development of a number of wellused e-resources for students: Making Group-Work Work, for instance, uses multiple media including short videos, audio commentaries and reflective exercises. [4] The obvious advantage of online dissemination is accessibility, while using electronic formats promotes the use of innovative approaches.

However, there were a number of issues raised here in relation to effectiveness. Partners working with distance-learning and mature students noted difficulties with access (using dial-up connections, for instance, or lack of technical experience). In some areas students claimed to find it easier to understand and remember information in a printed format. At the same time, there were comments on the expense of printing off paper copies of online resources: the consequent concern was that this might make financially-pressed students less likely to use resources directly disseminated in this way.

There were also implications for staff. While electronic resources can utilise technological innovations, these may be time-consuming and expensive to develop and require regular updating. Consequently, there is a need for staff to train in new technologies and to have the time to maintain resources once produced.

Online dissemination remains an easy and effective way to maximise accessibility and innovation in support resources, and e-resources are certainly popular with the majority of students. However LearnHigher's collaborative approach to research was able to illuminate some of the less-often heard corners of the student community, offering a useful reminder that learning development is never a 'one-size-fits-all' enterprise. 
The examples above represent a small sample of the issues discussed in the full report which also describes insights on the use of learning spaces funded by the CETL, on the role of learning developers generally, and on the interactions of subject academics with learning development. The LearnHigher network thus offers a microcosm of preferred practice in the learning development community: eschewing a narrow focus in favour of a more inclusive understanding, and valuing the professional expertise generated through the fruitful generosity of collaboration.

\section{Notes}

1. For more information on LearnHigher, including a list of partner institutions and learning areas, and free access to a wide range of research-informed learning development resources, see the website at www.learnhigher.ac.uk.

2. A full report on this project titled Taking an Overview of LearnHigher Research will be published in Spring 2009, and will be available to order or download via the LearnHigher website (www.learnhigher.ac.uk).

3. The LearnHigher Referencing website can be found at http://www.learnhigher.org.uk/.

4. Making Group-Work Work was developed collaboratively by LearnHigher partners at the Universities of Bradford and Leeds, and Brunel University and can be found at www.learnhighergroupwork.com/. Other electronic resources include Notemaker, developed at London Metropolitan University (http://learning.londonmet.ac.uk/TLTC/learnhigher/notemaker/). All of the resources developed so far can be accessed through the LearnHigher website (see n.1).

\section{References}

Neville, C. (2007) The Complete Guide to Referencing and Avoiding Plagiarism. Maidenhead: Open University Press.

Kim Shahabudin is a member of the University Study Advice team and LearnHigher Research Officer at the University of Reading. 